\title{
THE VOLUME OF A SLIGHTLY CURVED SUBMANIFOLD IN A CONVEX REGION
}

\author{
B. V. DEKSTER ${ }^{1}$
}

\begin{abstract}
Let $T$ be a compact convex region in an $n$-dimensional Riemannian space, $k_{s}$ be the minimum sectional curvature in $T$, and $\kappa>0$ be the minimum normal curvature of the boundary of $T$. Denote by $P^{v}(\xi)$ a $\nu$-dimensional sphere, plane or hyperbolic plane of curvature $\xi$. We assume that $k_{s}, \kappa$ are such that on $P^{2}\left(k_{s}\right)$ there exists a circumference of curvature $\kappa$. Let $R_{0}=R_{0}\left(\kappa, k_{s}\right)$ be its radius. Now, let $Q$ be a convex (in interior sense) $m$-dimensional surface in $T$ whose normal curvatures with respect to any normal are not greater than $\chi$ satisfying $0 \leqslant \chi<\kappa$. Denote by $L_{\chi}$ the length of a circular arc of curvature $\chi$ in $P^{2}\left(k_{s}\right)$ with the distance $2 R_{0}$ between its ends. We prove that the volume of $Q$ does not exceed the volume of a ball in $P^{m}\left(k_{s}-(n-m) \chi^{2}\right)$ of radius $\frac{1}{2} L_{\chi}$. These volumes are equal when $T$ is a ball in $P^{n}\left(k_{s}\right)$ and $Q$ is its $m$-dimensional diameter.
\end{abstract}

1. Statement of the result. Let $M$ be an $n$-dimensional Riemannian space, $n \geqslant 2$, of regularity class $C^{4}$. Throughout this paper, it will be assumed that manifolds, their edges, surfaces, mappings etc. all are of class $C^{4}$ without a special mention. We consider in $M$ a connected region that has a compact closure $T$ and is bounded by a nonempty hypersurface $\Gamma$. We suppose that all the normal curvatures of $\Gamma$ on the side of the interior normal are not less than some positive number $\kappa$. (Hence, $\Gamma$ is two-sided and $T$ is situated on one side of $\Gamma$.) Suppose that in the compact region $T$ the sectional curvatures $\geqslant k_{s}>$ - $\kappa^{2}$. In such a case we will call $T$ normal.

Denote by $P^{m}\left(k_{s}\right)$ an $m$-dimensional sphere, Euclidean or hyperbolic space of curvature $k_{s}$. It was remarked in $[3, \S 1]$, that on $P^{2}\left(k_{s}\right)$ there exists a circle $T_{0}$ whose circumference $\Gamma_{0}$ has geodesic curvature $\kappa$ if and only if $k_{s}>-\kappa^{2}$.

Denote by $Q$ a connected $m$-dimensional Riemannian manifold, $2 \leqslant m<$ $n$, with an edge (possibly empty) $\partial Q \subset Q$. We call $Q$ convex if for any $p$, $q \in Q, p \neq q$, there exists a minimal geodesic with the ends $p, q$.

An isometric immersion $i: Q \rightarrow T$ will be called $\chi$-curved, $\chi \geqslant 0$, if the curvature of the image of any geodesic in $Q$ under the mapping $i$ does not exceed $\chi$.

Let $\chi<\kappa$ and $i: Q \rightarrow T$ be a $\chi$-curved immersion. It follows immediately from the corollary of Theorem 1 in $[3, \S 1]$, that any geodesic in $Q$ is not longer than the length $L_{\chi}$ of a circular arc in the circle $T_{0}$ which has geodesic

Received by the editors August 4, 1976.

AMS (MOS) subject classifications (1970). Primary 53C40.

'Supported by NRC of Canada Grants awarded to Professors H. S. M. Coxeter, W. H. Greub, P. Scherk, D. K. Sen and J. R. Vanstone. 
curvature $\chi$ and whose ends are opposite points of $\Gamma_{0}$. Therefore, in fact,

$$
\partial Q \neq \varnothing \text {. }
$$

If $Q$ is convex, then obviously the diameter $D$ of $Q$ satisfies

$$
D \leqslant L_{\chi}
$$

We prove the following

THEOREM. Let a manifold $Q$ be convex, $\chi<\kappa$ and $i: Q \rightarrow T$ be a $\chi$-curved immersion. Then (i) $Q$ is a compact manifold with a nonempty edge and (ii) volume of $Q$ does not exceed the volume of a ball $B$ of radius $\frac{1}{2} L_{x}$ in $P^{m}\left(k_{s}-(n-m) \chi^{2}\right)$.

REMARKs. 1. The statement (i) has already been proved in the preceding remarks (see (1.1) and (1.2)).

2. $Q$ and $B$ have equal volumes when $T$ is a ball in $M=P^{n}\left(k_{s}\right)$ and $Q$ is an $m$-dimensional diameter of $T$. (Then $\chi=0$.)

3. If $P^{m}\left(k_{s}-(n-m) \chi^{2}\right)$ for $m=1$ is regarded as $R^{1}=R$, then this theorem generalizes the corollary in $[3, \S 1]$.

4. If $Q$ is not convex, then its volume can be arbitrarily large.

5. If $\kappa \leqslant 0$ or $k_{s} \leqslant-\kappa^{2}$, then, in such a region, the submanifold $Q$ can have arbitrarily large diameter and volume, even if the immersion $i$ is 0 -curved.

6. The smoothness requirement $\left(C^{4}\right)$ is not really essential. It only allows for the use of results in [1], [2], [3] and could be reduced to $C^{2}$ by means of a suitable approximation.

7. It is easy to see that $B$ is not more than a semisphere of $P^{m}\left(k_{s}-(n-\right.$ $\left.m) \chi^{2}\right)$ when $k_{s}-(n-m) \chi^{2}>0$.

8. If $\frac{1}{2} L_{\chi}$ in this Theorem is replaced by $L_{\chi}$, then such a rougher estimate can be obtained in a simpler way: instead of the remote point $q$ defined in $\$ 2$, we can use then an arbitrary point of $Q$.

2. Proof of Theorem. In view of Remark 1, we need to prove only (ii).

LEMmA 1. Sectional curvatures of the manifold $Q \backslash \partial Q$ are not less than $k_{s}-(n-m) \chi^{2}$.

Proof. Let $q \in Q \backslash \partial Q$ and $\sigma$ be a 2-dimensional direction at the point $q$. Denote by $\sigma_{*}$ the image of $\sigma$ under the mapping $i_{* q}: Q_{q} \rightarrow M_{i(q)}$. Let $k(\sigma)$ and $k\left(\sigma_{*}\right)$ be the curvatures of the manifolds $Q$ and $M^{q}$ in the directions $\sigma$ and $\sigma_{*}$. From formula (9) in [4, point 3.7], we have that:

$$
k(\sigma)=k\left(\sigma_{*}\right)+\sum_{\mu=1}^{n-m}\left|\begin{array}{ll}
l_{\mu}(u, u) & l_{\mu}(u, v) \\
l_{\mu}(u, v) & l_{\mu}(v, v)
\end{array}\right|
$$

where $u, v \in \sigma$ are orthogonal unit vectors and $l_{\mu}(\cdot, \cdot)$ is the second quadratic form of $Q$ with respect to a unit normal $N_{\mu}, \mu=1,2, \ldots, n-m$, satisfying $N_{\mu} \perp N_{\nu}$ when $\mu \neq \nu$.

Denote by $u_{\mu}, v_{\mu}$ orthogonal unit vectors showing the priscipal directions of 
the form $l_{\mu}$ restricted to $\sigma$. Then

$$
\left|\begin{array}{ll}
l_{\mu}(u, u) & l_{\mu}(u, v) \\
l_{\mu}(u, v) & l_{\mu}(v, v)
\end{array}\right|=\left|\begin{array}{cc}
l_{\mu}\left(u_{\mu}, u_{\mu}\right) & 0 \\
0 & l_{\mu}\left(v_{\mu}, v_{\mu}\right)
\end{array}\right| \geqslant-\chi^{2} .
$$

It follows from the inequalities $k\left(\sigma_{*}\right) \geqslant k_{s}$ and (2.2) that $k(\sigma) \geqslant k_{s}-$ $(n-m) \chi^{2}$. Q.E.D.

We will denote by $\rho(\cdot, \cdot)$ the intrinsic distance between the sets in the region $T$, and by $a b$ we will denote a shortest path with the ends $a, b \in T$. We will also use the notation $a b$ for $\rho(a, b)$. A shortest path $a b$ always exists and it is a geodesic. This fact follows from an argument analogous to that contained in Lemma 2 and the remark after it in [1].

By compactness of $Q$, there exists a point $q \in Q$ such that $\rho(i(q), \Gamma)=$ $\max _{p \in Q} \rho(i(p), \Gamma)$. We will call $q$ the remote point of $Q$. Obviously, $i(q) \in$ int $T$ because otherwise $i(Q) \subset \Gamma$ and the image under the immersion $i$ of any geodesic in $Q$ has the curvature not less than $\kappa$. This is obviously impossible by the definition of $\chi$-curved immersion $i$, and by the fact that $\chi<\kappa$.

Let $b \in T$. A shortest path $b a, a \in \Gamma$, will be called $b$-projecting if $b a=$ $\rho(b, \Gamma)$.

Lemma 2. Let $q$ be a remote point of $Q$ and let $b=i(q)$. Denote by $\Omega \subset Q_{q}$ the set of directions (unit vectors) of the geodesic emanating from $q$ toward the interior of $Q^{2}$

Then for any $u \in \Omega$ there exists a b-projecting shortest path which forms an angle $\phi \leqslant \pi / 2$ with the vector $i_{*}(u)$.

The proof of Lemma 2 will be given in $\$ 3$. Meanwhile we continue with the proof of the theorem.

Obviously $\Omega$ is homeomorphic to an open $(m-1)$-dimensional circle when $q \in \partial Q$. Denote by $C$ the cut locus of $Q$ from the point $q$. Let $u \in \Omega$ and $z(u)>0$ be the minimum number such that $\exp _{q}(z(u) \cdot u) \in C \cup \partial Q \cdot z(u)$ exists since the set $C \cup \partial Q$ is closed (because $Q \backslash(C \cup \partial Q)$ is obviously open).

We want to show first that

$$
z(u) \leqslant \frac{1}{2} L_{\chi}, \quad u \in \Omega .
$$

By Lemma 2, there exists a $b$-projecting shortest path $b a$ which forms an angle $\phi \leqslant \pi / 2$ with the curve $g:[0, z(u)] \rightarrow T$ given by the formula

$$
g(l)=i\left(\exp _{q}(l u)\right) \text {. }
$$

Applying the inequality (1.4) in [3] (where $\bar{L}_{0}(\pi / 2)$ is obviously $\left.\frac{1}{2} L_{\chi}\right)$ to the curve $g$ of curvature $\leqslant \chi$ and the mentioned path $b a$ we obtain (2.3).

Let us put $\Phi=\cup l u$, where $u \in \Omega$ and $0<l<z(u)$. It is easy to see that

\footnotetext{
${ }^{2}$ We mean that all points of the geodesic sufficiently close to $q$ and different from $q$ lie in int $T$. If $q \notin \partial Q$, then $\Omega=S^{m}$.
} 
for any $p \in$ int $Q, p \neq q$, the minimum geodesic $q p$ emanates from $q$ toward the interior of $Q$. Therefore

$$
\Psi \stackrel{\text { def }}{=} \exp _{q}(\Phi)=Q \backslash(q \cup \partial Q \cup C)
$$

and the mapping $\exp _{q}$ restricted to $\Phi$ is one-to-one. By an argument contained in Lemma 8 in [2] we can see that the set $C$ has $m$-dimensional measure zero. Therefore

$$
V(Q)=V(\Psi)
$$

where $V(\cdot)$ means volume.

Let $\tilde{q}$ be the center of the ball $B$ in the space $\tilde{Q} \stackrel{\text { def }}{=} P^{m}\left(k_{s}-(n-m) \chi^{2}\right)$ and $j: Q_{q} \rightarrow \tilde{Q}_{\tilde{q}}$ be an isometry. Let us put

$$
f=\exp _{\tilde{q}} \circ j \circ \exp _{q}^{-1}: \Psi \rightarrow \tilde{Q} .
$$

By (2.3), the set $\tilde{\Psi} \stackrel{\text { def }}{=} f(\Psi) \subset B$ so that

$$
V(\tilde{\Psi}) \leqslant V(B) .
$$

On the strength of (2.5) and (2.7), the proof will be completed if we show that

$$
V(\Psi) \leqslant V(\tilde{\Psi})
$$

To show this it is enough to establish that the induced mapping $f_{*}: T \Psi \rightarrow$ $T \tilde{\Psi}$ does not decrease the length of tangent vectors. Really, if it is proved then the mapping $f$ does not decrease volumes since, for any point $p \in \Psi$, the mapping $f_{* p}: \Psi_{p} \rightarrow \tilde{\Psi}_{\tilde{p}}(\tilde{p} \stackrel{\text { def }}{=} f(p))$, like any linear transformation, can be reduced to expansions into $m$ pair-wise orthogonal directions and to orthogonal transformations.

Let $p \in \Psi$ and a vector $v \in \Psi_{p}$. We need to show that $\left|f_{*}(v)\right| \geqslant|v|$. Let the vectors $v=$ and $v^{\perp}$ be such that $v=v=+v^{\perp}, v^{=\perp v^{\perp}}$, and $v=$ is directed along the shortest path $p q$. It follows easily from the definition of $f$ that $f_{*}\left(v^{-}\right)$is directed along $\tilde{p} \tilde{q},\left|f_{*}\left(v^{=}\right)\right|=\left|v^{=}\right|$, and $f_{*}\left(v^{\perp}\right) \perp f_{*}\left(v^{=}\right)$. Since $f_{*}(v)=f_{*}\left(v^{=}\right)+f_{*}\left(v^{\perp}\right)$, it will be enough to prove that $\left|f_{*}\left(v^{\perp}\right)\right| \geqslant\left|v^{\perp}\right|$.

Let $p(t), t \in[0, \varepsilon]$, be a curve such that $p(0)=p, \dot{p}(0)=v^{\perp}$ and $q p(t)=$ $q p$. We put $\tilde{p}(t)=f(p(t))$. Obviously, $\tilde{q} \tilde{p}(t)=q p(t)=q p$, and $\Varangle \tilde{p} \tilde{q} \tilde{p}(t)=\Varangle$ $p q p(t)$. By Rauch comparison theorem we have: $|\dot{\tilde{p}}(0)| \geqslant|\dot{p}(0)|$, i.e. $\left|f_{*}\left(v^{\perp}\right)\right|$ $>\left|v^{\perp}\right|$. This completes the proof of the theorem.

3. Proof of Lemma 2. Suppose otherwise. That is suppose that some $u \in \Omega$ forms an angle $>\pi / 2$ with any $b$-projecting shortest path. We consider the curve $g$ given by (2.4). Let $l_{i \rightarrow \infty} 0, l_{i}>0$, and $b_{i}=g\left(l_{i}\right), i=1,2, \ldots$ Denote by $b_{i} a_{i}$ a $b_{i}$-projecting shortest path. By a simple compactness argument (selection of a subsequence), we may regard the sequence $b_{i} a_{i}$ as converging to a $b$-projecting shortest path $b a$.

We first want to prove the following statement

$(\alpha)$ there are no conjugate points on the segment $b a$.

Let us extend $b a$ beyond its end $a$ (as a geodesic) to a close point $d$ such 
that the extension ad is still a shortest path and $S \cap T=a$ where $S$ is the sphere of radius $a d$ with the center at $d$ (see the figure). If $(\alpha)$ is not true, then there exists an arc $\breve{b d}$ satisfying

$$
l(\breve{b d})<b a+a d
$$

where $l(\cdot)$ means the length. In what follows we will denote by $\div$ parts of the arc $\breve{b d}$. Let $e \in \breve{b d}, \breve{b e} \subset T, e \in \Gamma$ and let $h \in \breve{b d} \cap S$. We have:

$$
\begin{aligned}
& l(\breve{b e}) \leqslant l(\breve{b h}), \\
& l(\breve{h d}) \geqslant a d .
\end{aligned}
$$

Subtracting (3.3) from (3.1) we obtain that $l(\breve{b h})<b a$. Combining it with (3.2) we find that $l(\breve{b e})<b a$ which is impossible by definition of $b$-projecting shortest path $b a$. So, $(\alpha)$ has been proved.

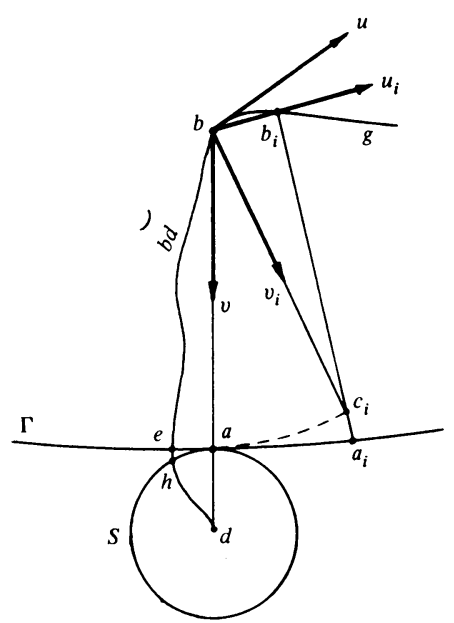

For sufficiently large $i$ the inequality $b b_{i}<b a \leqslant b a_{i}$ holds. Therefore on $b_{i} a_{i}$ there exists a point $c_{i}$ such that $b c_{i}=b a$. Let us connect $b$ and $c_{i}$ with a shortest path $b c_{i}$ and consider the triangle $b b_{i} c_{i}$ (see the figure). The direction $u_{i} \in \Omega$ of its side $b b_{i}$ converges to $u$. The relation $a_{i} \rightarrow a$ obviously implies $c_{i} \rightarrow a$. This fact together with $(\alpha)$ means that the direction $v_{i}$ of the side $b c_{i}$ converges to the direction $v$ of $b a$.

Let us regard the directions $u_{i}, v_{i}$ and the length $b b_{i}$ as three independent variables. As soon as $b c_{i}=b a$ does not depend on $i$ and because of $(\alpha)$, there exists, $\varepsilon>0$ such that the length $b_{i} c_{i}$ is a regular function $\lambda\left(u_{i}, v_{i}, b b_{i}\right)$ in the compact region $\sigma:\left|u_{i}-u\right| \leqslant \varepsilon,\left|v_{i}-v\right| \leqslant \varepsilon,\left|b b_{i}\right| \leqslant \varepsilon$. (Negative $b b_{i}$ means the displacement in the direction $-u_{i}$.) By Hadamard Lemma,

$$
\begin{aligned}
\lambda\left(u_{i}, v_{i}, b b_{i}\right)= & \lambda\left(u_{i}, v_{i}, 0\right)+\frac{\partial \lambda}{\partial b b_{i}}\left(u_{i}, v_{i}, 0\right) \cdot b b_{i} \\
& +\theta\left(u_{i}, v_{i}, b b_{i}\right) \cdot\left(b b_{i}\right)^{2}
\end{aligned}
$$

where the function $\theta$ is regular in $\sigma$ and therefore bounded. Let $C$ be such that $|\theta|<C$. For sufficiently small $\varepsilon$, the segment $b c_{i}$ being close to $b a$ does 
not contain conjugate points. Therefore $\partial \lambda\left(u_{i}, v_{i}, 0\right) / \partial b b_{i}=-\left\langle u_{i}, v_{i}\right\rangle$. Obviously, $\lambda\left(u_{i}, v_{i}, 0\right)=b a$.

Now, for large $i$, the point $\left(u_{i}, v_{i}, b b_{i}\right) \in \sigma$ and we get from (3.4) that

$$
b_{i} c_{i}>b a+b b_{i}\left(-\left\langle u_{i}, v_{i}\right\rangle-C \cdot b b_{i}\right)
$$

By our original contrary assumption, $\langle u, v\rangle\left\langle 0\right.$. Therefore $\lim _{i \rightarrow \infty}\left\langle u_{i}, v_{i}\right\rangle=$ $\langle u, v\rangle<0$. Since $b b_{i \rightarrow \infty} 0$, we find from (3.5) that $b_{i} c_{i}>b a$ for sufficiently large $i$. Consequently, $b_{i} a_{i}>b a$. But then the point $q$ ( $q \in$ $\left.i^{-1}(b)\right)$ is not remote. This contradiction proves the Lemma.

\section{BIBLIOGRAPHY}

1. B. V. Dekster, Estimates of the volume of a region in a Riemannian space, Math. USSR-Sb. 17 (1972), 61-87.

2. An inequality of the isoperimetric type for a domain in a Riemannian space, Math. USSR-Sb. 19 (1973), 257-274.

3. __ Estimates of the length of a curve, J. Differential Geometry 12 (1977), 99-115.

4. D. Gromoll. W. Klingenberg and W. Meyer, Riemannische Geometrie im Grossen, Lecture Notes in Math., vol. 55 Springer-Verlag, Berlin and New York, 1968.

Department of Mathematics, University of Toronto, Toronto, Ontario, Canada 